\title{
Iatrogenia en personas diagnosticadas de trastorno límite de la personalidad
}

\section{Iatrogenesis in people with a diagnosis of borderline personality disorder}

\author{
Cristina Rodríguez Cahill ${ }^{1,2}$, Gustavo Ruiz Llavero², David Martín Escudero ${ }^{2}$, \\ Pablo Garnelo Fernández ${ }^{2}$, Fernando Sánchez Rodríguez ${ }^{2}$, María Isabel Casares García ${ }^{2}$ \\ y Katherine Morales González ${ }^{3}$ \\ ${ }^{1}$ SERMAS, Hospital Universitario de Alcalá de Henares, España \\ ${ }^{2}$ Miembros del Grupo de trabajo de Trastornos de la Personalidad del Colegio de la Psicología de Madrid, España \\ ${ }^{3}$ SEHR. Consultoría y Psicoterapia, España
}

\begin{abstract}
Resumen: Existe en los psicoterapeutas una tendencia a sobreestimar los resultados de sus intervenciones además de infravalorar los riesgos a los que exponen a sus pacientes. Aunque la psicoterapia ha demostrado con creces su eficacia, conviene tener en cuenta que entre el 5 y el 10 por ciento de los pacientes empeoran durante el proceso psicoterapéutico y que tres de cada diez manifiestan cambios clínicamente poco significativos, de acuerdo a diferentes investigaciones. Este texto se propone revisar la diversidad del daño que se puede derivar de la psicoterapia con pacientes diagnosticados de un trastorno límite de la personalidad. En las personas con este diagnóstico se incrementa el riesgo de iatrogenia como resultado de la activación de su sistema de apego. El artículo hace un recorrido por las fuentes de efectos perjudiciales finalizando con algunas recomendaciones para la disminución del riesgo que subyace en la psicoterapia con estos pacientes.

Palabras claves: Iatrogenia, riesgo psicoterapéutico, daño psicoterapéutico, trastorno límite de la personalidad, trastorno identitario.
\end{abstract}

\begin{abstract}
Psychotherapists tend to overestimate the positive results of the therapy they provide, while also underestimating the risks that their patients are exposed to due to praxis. Although psychotherapy has shown over and again its efficacy on the whole, it is worth
\end{abstract}

Cristina Rodríguez Cahill es Psicóloga en el Servicio Madrileño de Salud - SERMAS, en el Hospital Universitario de Alcalá de Henares.

Gustavo Ruiz Llavero, David Martín Escudero, Pablo Garnelo Fernández, Fernando Sánchez Rodríguez, María Isabel Casares García son

Psicólogos, miembros del Grupo de trabajo de Trastornos de la Personalidad del Colegio Oficial de la Psicología de Madrid.

Katherine Morales González es psicóloga en SEHR - Servicios especializados en recursos y gestión humana.

Para citar este artículo: Rodríguez Cahill, C., Ruiz Llavero, G., Martín Escudero, D., Garnelo Fernández, P., Sánchez Rodríguez, F., Casares

García, M. I. y Morales González, K. (2021). Iatrogenia en personas diagnosticadas de trastorno límite de la personalidad. Clínica Contemporánea,

12(3), Artículo e22. https://doi.org/10.5093/cc2021a21

La correspondencia sobre este artículo debe enviarse a Cristina Rodríguez Cahill al e-mail: criscahill@yahoo.es

(cc) BY-NC-ND Este es un artículo Open Access bajo la licencia CC BY-NC-ND. 
underlining that $5-10 \%$ of the patients worsen during the psychotherapy process, and $30 \%$ present insignificant clinical change, according to different research studies. In this article, we aim to review the diversity of the harm that can result from psychological intervention when working with patients diagnosed with borderline personality disorder. People with this diagnosis are highly prone to suffer harm during psychological intervention due to the activation of their attachment system. In this article, the myriad sources of these harmful effects are reviewed, and recommendations are provided for minimizing the risk inherent to psychotherapy in these patients.

Keywords: Iatrogenesis, psychotherapy risk, psychotherapy harm, borderline personality disorder, identity disorder

El principal objetivo de este artículo es sintetizar el conocimiento actual relevante sobre los efectos potencialmente dañinos de las intervenciones psicoterapéuticas en personas con un diagnóstico de trastorno de la personalidad, especialmente cuando existe un diagnóstico de trastorno límite. La psicoterapia sola, o en combinación con otras intervenciones, constituye el tratamiento de primera elección de un gran número de trastornos mentales, como es el caso de los trastornos de personalidad. Por esta razón, el estudio de la iatrogenia derivada de la psicoterapia realizada con estos pacientes resulta un problema de gran relevancia clínica, social y ética.

También hemos querido compartir las reflexiones elaboradas sobre este tema por los miembros del grupo "Trastornos de la Personalidad" del Colegio Oficial de la Psicología de Madrid a lo largo de nuestro trabajo conjunto.

\section{La iatrogenia en psicoterapia}

Con frecuencia los psicoterapeutas sobreestiman los efectos positivos de sus terapias y obvian los riesgos a los que exponen a los pacientes con sus intervenciones. Aunque la psicoterapia ha probado su efectividad en términos generales, también se sabe que un 5-10\% de los pacientes empeoran durante el proceso y más de un $30 \%$ presentan cambios que no son clínicamente significativos (Lambert y Ogles, 2004). Existen pocos estudios en Europa sobre los resultados negativos atribuidos a la psicoterapia. En el caso de Reino Unido, se estima que 1 de cada 20 personas de quienes asisten a psicoterapia va a experimentar efectos negativos duraderos (Crawford et al., 2016). Jarrett (2008) menciona que aproximadamente 1 de cada 10 clientes que asisten a terapia empeora y que, pese a esa evidencia, los terapeutas parecen ignorar los efectos secundarios y dañinos que pueden acompañar a la psicoterapia. Así, cita el estudio que Boisvert realizó en 1999 (tesis doctoral no publicada) donde la mayoría de los psiquiatras consultados ignoraban la posibilidad de provocar daños derivados de la psicoterapia.

En España, se sabe que una de cada cuatro personas de la población ha hecho uso alguna vez de los servicios de salud mental públicos y que la media de espera puede superar los 200 días en alguna Comunidad, lo que probablemente redunde en un aumento del malestar (Sánchez Becerril, 2019). A esto se suma que la frecuencia de citas que reciben los pacientes en este contexto suele ser mensual o bimensual, frecuencia muy por debajo de las recomendaciones hechas por diferentes guías y protocolos clínicos para una psicoterapia. En el caso de Andalucía, el defensor del pueblo realizó un estudio donde se concluía lo siguiente: el descontento y la disconformidad con la asistencia recibida es tal, en algunos casos, que pacientes y familiares aseguran que el trato recibido ha llegado a empeorar la sintomatología del paciente (Infocop, 2013).

A medida que se consolidan las terapias especializadas manualizadas, y que los estudios de caso, los ECA (Ensayo Controlado y Aleatorizado, del inglés Randomized controlled Trial [RCT]) y las Guías Clínicas se convierten en base importante del conocimiento para considerar qué y cuánta terapia se va a necesitar, ha 
quedado atrás la percepción de la psicoterapia como un placebo para el sufrimiento o que su éxito se debía a la recuperación espontánea (Eysenck, 1965) y se ha incrementado la preocupación por limitar sus efectos perjudiciales, como sucede con los tratamientos médicos.

Desde hace décadas se habla del problema de los efectos adversos de las terapias psicológicas pero la investigación sobre sus causas y su prevención no ha evolucionado suficientemente. A la hora de identificar cuáles pueden ser los efectos perjudiciales derivados de la psicoterapia o de alguno de sus componentes, la investigación se vuelve confusa por la diversidad de definiciones y por la cantidad de términos que se utilizan para hacer referencia a sus efectos indeseables; exacerbación de síntomas, efectos negativos, fracaso del tratamiento, deterioro clínico, resultado negativo, reacción negativa a la terapia, daño terapéutico, psicoiatrogenia, seguridad del paciente, entre otros (Parry, Crawford y Duggan, 2016). Esta dispersión de expresiones se acompaña de una gran cantidad de quejas de pacientes que pueden encontrarse a lo largo de Internet de la atención recibida por los profesionales de la salud mental y que nos alerta de que las posibilidades de daño son amplias. En este artículo se propone usar el término iatrogenia para hacer referencia a los efectos indeseables del tratamiento o de la ausencia del mismo. Se hace necesario un marco de referencia sólido que permita un avance en este campo. Impulsar la investigación y el debate en relación a los efectos indeseables de las terapias psicológicas es importante, especialmente en pacientes con patología grave, como los diagnosticados de un trastorno de personalidad.

Resulta una tarea ardua establecer relaciones causa-efecto sobre el impacto negativo de un tratamiento psicológico. En la mayoría de los casos estos aparecen de manera menos evidente que los que pueden producir otras intervenciones en el ámbito de la salud. Los psicoterapeutas deben reconocer la probabilidad de provocar inadvertidamente efectos negativos en el paciente durante el tratamiento psicológico, aceptar que en el ámbito de la salud mental siempre existe esta posibilidad y que no siempre está vinculada de manera explícita a una intervención inadecuada (Boisvert y Faust, 2002). Sería deseable ser más conscientes y reflexionar sobre cómo evaluar y gestionar aquellos momentos que pueden tener un impacto negativo en el proceso terapéutico o en el bienestar del paciente (Castonguay et al., 2010). Nuestro grupo propone distinguir entre dos posibles tipos de iatrogenia puesto que los efectos dañinos también pueden emerger de la omisión o insuficiencia de tratamiento, es decir, de la ausencia de intervención en el momento terapéutico apropiado. Recomendamos considerar como iatrogenia negativa aquella relativa a la ausencia de intervención adecuada y iatrogenia positiva como aquella que sigue a una acción perniciosa para el paciente.

\section{Tabla 1. Subtipos de Iatrogenia}

\section{IATROGENIA POSITIVA}

Efecto dañino provocado por una acción que resulta perniciosa para el paciente
IATROGENIA NEGATIVA

Omisión 0 insuficiencia de una intervención en un momento terapéutico apropiado que implica un empeoramiento o que obstaculiza la mejoría esperable del paciente con tratamiento

Evitar el daño en nuestras intervenciones psicoterapéuticas no impide la iatrogenia, pues simplemente no ayudar o prestar un servicio insuficiente también puede tener efectos iatrogénicos. Esto genera en los pacientes y sus familiares la creencia de que las intervenciones no funcionan, que tienen pocas posibilidades de cambio, provocando un bajo nivel de autoeficacia que afectará posibles tratamientos futuros (Jones, 2007).

El objetivo fundamental del tratamiento debe ser maximizar los beneficios y minimizar el daño. Eliminar completamente cualquier efecto negativo del tratamiento tampoco es realista y solo sería posible suspendiendo el mismo, lo cual, dado el balance positivo general de los resultados del tratamiento, en última instancia, haría un daño considerablemente mayor al paciente (Boisvert y Faust, 2002). En este artículo se analizan los factores que convierten los efectos negativos del tratamiento en iatrogenia y cómo pueden ser subsanados. Los terapeu- 
tas en ocasiones se equivocan y esto no siempre implica un daño en el paciente; es más, en ocasiones puede ser reconducido como una oportunidad para entender mejor al paciente y para mentalizar sobre lo sucedido.

Lo que distingue un efecto negativo del tratamiento de un tratamiento iatrogénico tiene que ver con el balance final entre efectos deseables y efectos perniciosos, así como la resolución de los efectos negativos por parte del profesional, si es que esto es posible.

\section{La iatrogenia en pacientes con un diagnóstico de trastorno de la personalidad}

Las terapias psicológicas tienen un alto riesgo iatrogénico especialmente en personas con grave patología identitaria, especialmente con patología borderline, en la que nos centraremos en este artículo. Zanarini y Frankenburg (2007) describen la "reacción tóxica" como un efecto negativo específico del tratamiento psicológico sobre algunos de estos pacientes, pero también señalan que no se conocen todavía sus mecanismos de actuación. Según Fonagy y Allison (2014), las personas con este diagnóstico, especialmente aquellas que sufren un trastorno límite, son más susceptibles de sufrir daños de las intervenciones debido a la hiperactivación de su sistema de apego, lo que se produce al iniciar una psicoterapia. Con frecuencia, las intervenciones psicológicas estimulan las necesidades de apego de estos pacientes, pero no aportan la estructura necesaria, ni se realizan en las mínimas condiciones requeridas para trabajar sus dificultades. Esto lleva a muchos pacientes a abandonar el tratamiento o a una escalada de consultas en los servicios de urgencias o, en el peor de los casos, a severas autoagresiones. A continuación, se recoge una adaptación de la clasificación de Parry, Crawford y Duggan (2016), para exponer las variables que influyen en que la terapia con estos pacientes pueda ser perjudicial:

- Relación terapéutica: Perjuicios provocados por la relación entre terapeuta y paciente, así como rupturas de la alianza terapéutica que no son reparadas.

- Terapeuta: Uso inadecuado de un tipo de terapia; mala aplicación de una técnica terapéutica; desconocimiento del modelo de terapia que se está utilizando; inexperiencia con el tipo de trastorno al que se enfrenta; aspectos patológicos de la personalidad del terapeuta.

- Paciente: Riesgos asociados a la activación del apego hacia el terapeuta en pacientes con reducida capacidad de mentalización, como es el caso en algunos trastornos de personalidad; estado de hipervigilancia epistémica que dificulta el aprendizaje social de estos pacientes y, por tanto, el aprendizaje en un encuentro psicoterapéutico.

- Contratransferencia: Dificultades en la relación terapéutica evocadas por la transferencia que es actuada por el paciente o por aspectos no elaborados del terapeuta, como la que puede darse en situaciones donde el terapeuta se siente desbordado o removido por el tipo de información que comparte el paciente.

- Intervención técnica: Riesgos asociados a técnicas específicas o a la mala aplicación de las mismas. Como ejemplo, podemos señalar el caso de aprendizajes maladaptativos por parte de los miembros de un grupo en una intervención grupal.

- Institución: La organización dentro de la cual se enmarca el trabajo de los terapeutas tiene un efecto relevante en los pacientes, aunque habitualmente imperceptible. Cuando el profesional trabaja con demasiados casos y la institución no cuida ni sostiene a los profesionales, independientemente de los requisitos necesarios para una intervención o de la cualificación profesional específica necesaria, la intervención iatrogénica es un riesgo altamente probable.

El perjuicio que se puede infligir a estos pacientes abarca todo el proceso de atención, desde la prevención hasta el tratamiento, pasando por el proceso diagnóstico. Se hace fundamental conocer el daño que se puede generar en todos los momentos clínicos relevantes y tener en consideración las variables de los terapeutas, de los pacientes y del contexto para poder estimar con precisión el riesgo y los beneficios de comenzar un tratamiento psicoterapéutico con un paciente con diagnóstico de trastorno de la personalidad. El resto de este artículo se dividirá en dos grandes apartados: La "Intervención iatrogénica" y la "Gestión iatrogénica" para distinguir los efectos nocivos sobre los pacientes que son generados en el contexto de la relación con un terapeuta o equipo terapéutico, de aquellos daños provocados por la incorrecta gestión y organización de los servicios de salud mental (Rodríguez Cahill, 2015). 


\section{Figura 1.}

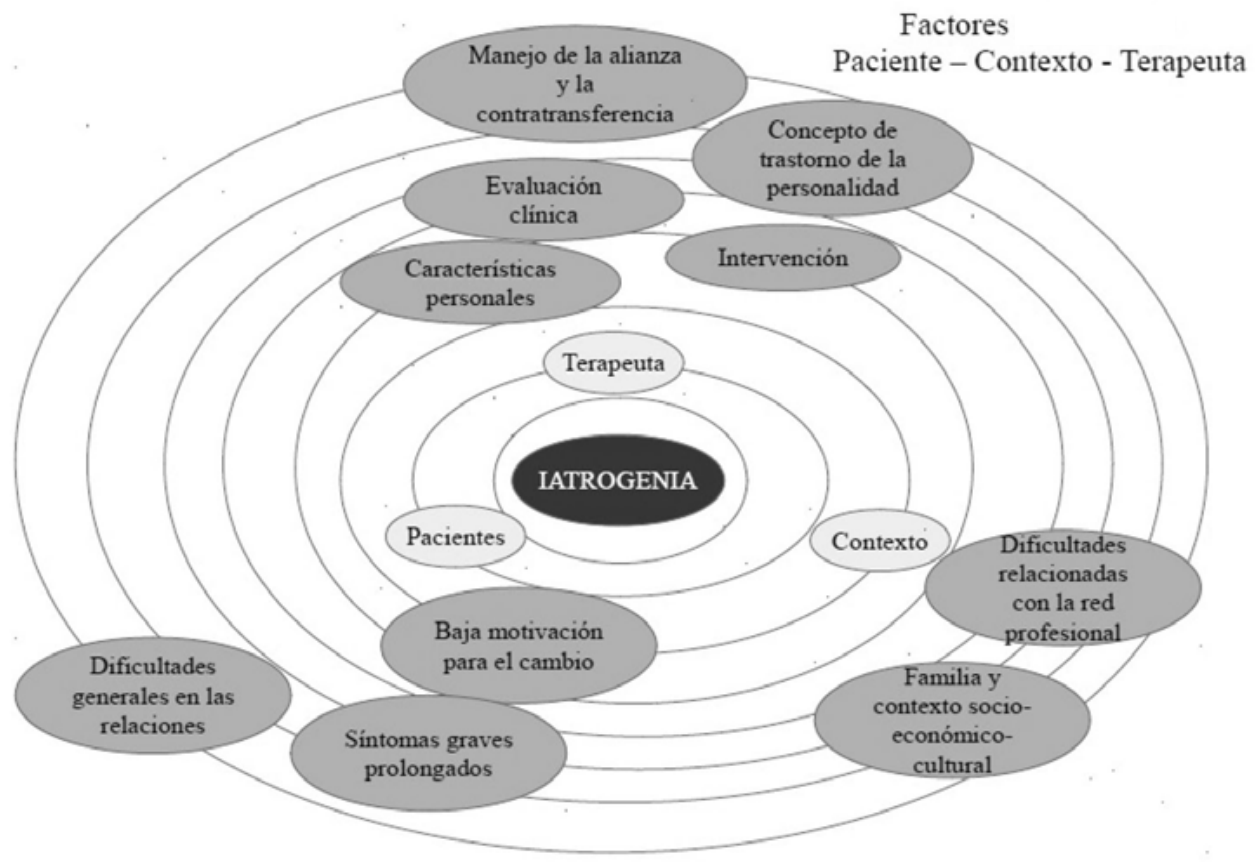

A continuación, se profundizará en las intervenciones iatrogénicas más frecuentes que cometen los terapeutas y los equipos de salud mental, así como los factores que correlacionan con resultados negativos a la hora de intervenir con personas con un trastorno de personalidad. Dentro del epígrafe "Intervención iatrogénica" se analizarán factores relacionados con los terapeutas, con los pacientes y con el contexto.

\section{Intervención iatrogénica}

\section{Factores relacionados con los terapeutas}

Concepto de trastorno de personalidad. La propia conceptualización de este diagnóstico, y los estereotipos y prejuicios asociados al mismo pueden resultar iatrogénicos. Chartonas y su equipo (2017) replicaron un estudio realizado en 1988 por Lewis y Appleby, donde se demostraba que los psiquiatras mantenían actitudes negativas hacia pacientes con un diagnóstico de trastorno de personalidad, con la expectativa de que la actitud hacia este tipo de pacientes habría mejorado en las últimas décadas. Estos autores evaluaron y compararon las actitudes de 166 psiquiatras en formación hacia pacientes con diagnóstico de trastorno límite de personalidad y hacia pacientes con diagnóstico de depresión. Los resultados volvieron a señalar que los profesionales mantenían una actitud más negativa hacia los pacientes con patología identitaria. Además, en el análisis factorial se encontró que los psiquiatras sentían que el trabajo tenía menos sentido cuando trataban con los pacientes con un diagnóstico de trastorno de personalidad.

El diagnóstico entendido como una mera etiqueta puede resultar estigmatizador e incluso limitar de forma importante el cambio en estos pacientes. Desde esta postura, la sobremedicalización y la infantilización de estas personas por parte de los profesionales de salud mental pueden llevar a una cronificación, y hasta una perpetuación de sus dificultades. 
Evaluación clínica. La nosología en salud mental es cambiante en función del momento histórico y de los consensos a los que llegan los profesionales. Las etiquetas diagnósticas no son realidades sino clasificaciones que nos aportan un lenguaje común a los profesionales centrado meramente en los síntomas. La última clasificación DSM-5 incluye una aproximación más dimensional a la clasificación de los trastornos de personalidad, pero todavía insuficiente. Las etiquetas diagnósticas, si no van acompañadas de un enfoque comprensivo de las dificultades del individuo, implican un alto riesgo de cosificar y estigmatizar el sufrimiento. Sobre la utilidad de los diagnósticos categoriales subsiste gran controversia. Existen evidencias que sugieren que utilizar diagnósticos psiquiátricos y modelos teóricos asociados implica una peor evolución clínica y peor adaptación social (Timimi, 2014). La utilidad de los diagnósticos descriptivos como los que aportan los sistemas DSM y CIE son, en ocasiones, de escasa ayuda para el tratamiento, y algunos estudios consideran que el acuerdo entre evaluadores a la hora de diagnosticar algunos trastornos (trastorno depresivo mayor y trastorno de ansiedad generalizada, entre otros) son muy reducidos y cercanos a la "casualidad" (Freedman et al, 2013, citado en Timimi, 2014). Los conceptos de funcionamiento y estructura psíquica han permitido la superación del dilema dimensiones-rasgos (Semerari y Dimaggio, 2008). Cuando se evalúa la personalidad deben ser valoradas en profundidad tanto la estructura psíquica como el funcionamiento actual y pasado. Entender el funcionamiento intrapsíquico e interpersonal de estas personas es fundamental para un abordaje terapéutico adecuado. En resumen, evaluar los aspectos metapsicológicos profundos de estos pacientes, independientemente del modelo comprensivo de referencia, permite a los profesionales tener un modelo factible del funcionamiento mental que oriente las intervenciones (Rodríguez Cahill, 2015). Por ejemplo, el diagnóstico estructural diseñado por Otto Kernberg permite una evaluación profunda de la patología identitaria que aporta gran riqueza para el tratamiento.

En esta fase, las principales causas de iatrogenia en estos pacientes suelen ser el manejo de modelos diagnósticos reduccionistas sin un modelo comprensivo de referencia, obviando la patología identitaria, los errores diagnósticos, sobrediagnosticando resistencias del paciente y no dedicando tiempo suficiente para una buena evaluación. Con relación al debate sobre si ofrecer o no el diagnóstico al paciente, cabe enfatizar que el diagnóstico en sí mismo es de poca utilidad sin una comprensión biográfica y psicológica profunda, más cercana a una formulación. Sin embargo, en ocasiones el diagnóstico es útil para estos pacientes o para sus familiares, pues transmite una sensación de que su sufrimiento es algo conocido. En otras ocasiones, sin embargo, el diagnóstico limita y preocupa al paciente. En este sentido, puede ser útil el paradigma de las decisiones compartidas que se está extendiendo en la práctica clínica, a la hora de plantearse si dar o no un diagnóstico.

A la hora de establecer el plan de tratamiento, debe optarse por su adecuación a la naturaleza y severidad del trastorno y a las características particulares del paciente. Esto se conoce como selección terapéutica diferencial, y supone el establecimiento de criterios que responden a las siguientes preguntas: qué tratamientos son efectivos para los trastornos de personalidad, para qué tipo de pacientes son mejores un tipo u otro, y bajo qué circunstancias. Anthony Roth y Peter Fonagy, en su libro What for whom? (2005), hicieron una revisión de los estudios realizados en diferentes patologías para dar respuesta a esta pregunta. Se apuesta por la especialización como se demuestra en numerosas investigaciones donde se comparan los resultados de una terapia específica frente al tratamiento habitual [TAU, Treatment As Usual]. En el tratamiento de personas con un trastorno de personalidad límite los abordajes específicos suelen obtener los mejores resultados, mientras que tras dos o tres años de tratamiento habitual, los resultados revelan cómo se mantienen los desajustes en satisfacción global, empleo, funcionamiento general y adaptación social (Lieb et al, 2004).

Intervención. El error más habitual a la hora de tratar a personas con problemas identitarios es priorizar el tratamiento farmacológico por encima del psicoterapéutico, siendo un problema frecuente la medicalización excesiva en estos pacientes. La revisión de las principales guías clínicas señala que el tratamiento nuclear para esta patología es el psicoterapéutico (Gunderson y Gabbard, 2002). Sin embargo, el tratamiento mayoritario que reciben las personas con este diagnóstico es el farmacológico.

Otro de los grandes riesgos en el tratamiento de personas con un diagnóstico de trastorno de personalidad es recibir tratamientos poco planificados, inconsistentes, improvisados y guiados por la contratransferencia de los profesionales. En este sentido, se daña frecuentemente a los pacientes o a sus familiares mediante la ausencia 
de una buena evaluación, intervenciones no enmarcadas dentro de un Plan Individual de Tratamiento (PIT), falta de coordinación entre los diferentes profesionales y espacios de elaboración y supervisión inexistentes. Cada vez que un paciente se pone en tratamiento, utiliza una opción terapéutica y si no se beneficia de ella, aumenta la desesperanza con relación a la posibilidad de cambio. Dado que estos pacientes sufren de forma desmesurada, suelen volver a demandar tratamiento a través de otros cauces o en otros contextos, convirtiendo a estas personas en los nuevos habitantes de la "puerta giratoria" del sistema de salud (Rodríguez Cahill, 2015).

Hay terapias que resultan efectivas a largo plazo, mientras que a corto plazo aumentan la sintomatología en los pacientes. Este hecho dificulta la intervención ya que, al haber un exacerbamiento de los síntomas, el terapeuta puede abandonar un tratamiento que a largo plazo es efectivo, generando efectos negativos sobre el paciente. Algunos pacientes diagnosticados con trastorno de la personalidad tienen serias dificultades para conectar con sus emociones y durante el proceso terapéutico, el comenzar a sentir emociones disfóricas por primera vez puede simbolizar una posibilidad de cambio para el paciente (Crown, 1983).

A continuación, se confecciona un listado de intervenciones posiblemente iatrogénicas con pacientes diagnosticados de trastorno límite de la personalidad:

- Llevar a cabo tratamientos no específicos para esta patología e inconsistentes.

- La falta de competencias o formación para trabajar con estos pacientes.

- Indicar psicoterapias o intervenciones que no proceden.

- Realizar intervenciones extemporáneas (no llevadas a cabo en un buen momento del paciente). Los errores de timing suelen deberse a la impaciencia del terapeuta a la hora de intervenir buscando empujar el proceso. Un ejemplo de esto podría ser llevar a cabo intervenciones del trauma demasiado tempranas, sin antes estar bajo control los síntomas que suponen un riesgo para el paciente (Van der Hart, Nijenhuis y Steele, 2008).

- Las pseudopsicoterapias (Rodríguez Cahill, 2015) que se definen como aquellas intervenciones que se encuadran como terapias psicológicas, pero no cumplen las condiciones mínimas imprescindibles para su realización, como, por ejemplo, una escasa frecuencia de citas.

- No atender la comorbilidad o, por el contrario, obviar la patología identitaria de base de otra patología y solo tratar los síntomas. Por ejemplo, una adicción o un trastorno de la conducta alimentaria.

- Mantener una visión reduccionista sin incluir la perspectiva sistémica y relacional de la patología identitaria.

- Expectativas irrealistas de éxito y competencia profesional instantánea, creencia de que el éxito depende únicamente de la correcta aplicación de la técnica y procedimientos aprendidos.

- Exceso de confianza o fuerte ansiedad e inseguridad en las propias capacidades del terapeuta, asumiendo toda la responsabilidad por el cambio del paciente.

- Miedo a la confrontación, dificultad en el manejo de la relación y del trabajo con el aquí y ahora de la sesión.

- Destruir la relación terapéutica al valorar negativamente algún aspecto del paciente (Campell, 1992, citado en Boisvert y Faust, 2002).

- La devaluación de la evidencia científica (Grove y Meehl, 1996, citado en Lilienfeld, Lynn y Lohr, 2015).

- La sobrepatologización del paciente, atribuir una cantidad mayor de psicopatología de la que existe en realidad (Furman y Abola, 1989, citado en Boisvert y Faust, 2002).

- Ingresos en urgencias recurrentes y ausencia de un plan de crisis. La investigación sugiere que las personas con antecedentes en servicios de urgencias psiquiátricas presentan un mayor riesgo de suicidio. Sin embargo, no está claro si este vínculo es atribuible a factores individuales del paciente o a efectos iatrogénicos de los propios servicios en algunos casos (Sinnaeve et al., 2018). Otro estudio reciente de pacientes con diagnóstico TLP, atribuye un impacto negativo de los ingresos y relaciona la asistencia a servicios de urgencias con un incremento en el riesgo de suicidio (Coyle et al., 2018).

En el tratamiento de personas con un diagnóstico de trastorno límite de personalidad, como en la psicoterapia en general, es necesario prestar atención a los factores comunes. Muchos tipos de terapias diferentes han demostrado una eficacia similar para tratar a estos pacientes, por lo tanto, cabría reflexionar sobre qué tienen en común. 
En psicoterapia, el veredicto del pájaro $D O D O$ representa metafóricamente la idea de que diversos enfoques obtienen resultados equivalentes. Desde esta premisa, se ha postulado que lo que prevalece en la eficacia de los tratamientos son los factores comunes más que los componentes específicos de las diferentes psicoterapias. Con relación al tratamiento de los trastornos de personalidad, se señala que las psicoterapias eficaces comparten las $3 \mathrm{C}$ : Consistencia, coherencia y continuidad, aspectos relevantes para el tratamiento de estos pacientes (Bateman et al., 2017). También apunta Rodríguez Cahill (2015) que el factor común de los tratamientos eficaces para trastornos de personalidad es que son programas de tratamiento intensivos, co-

Figura 2. Imagen "Gato de Cheshire"

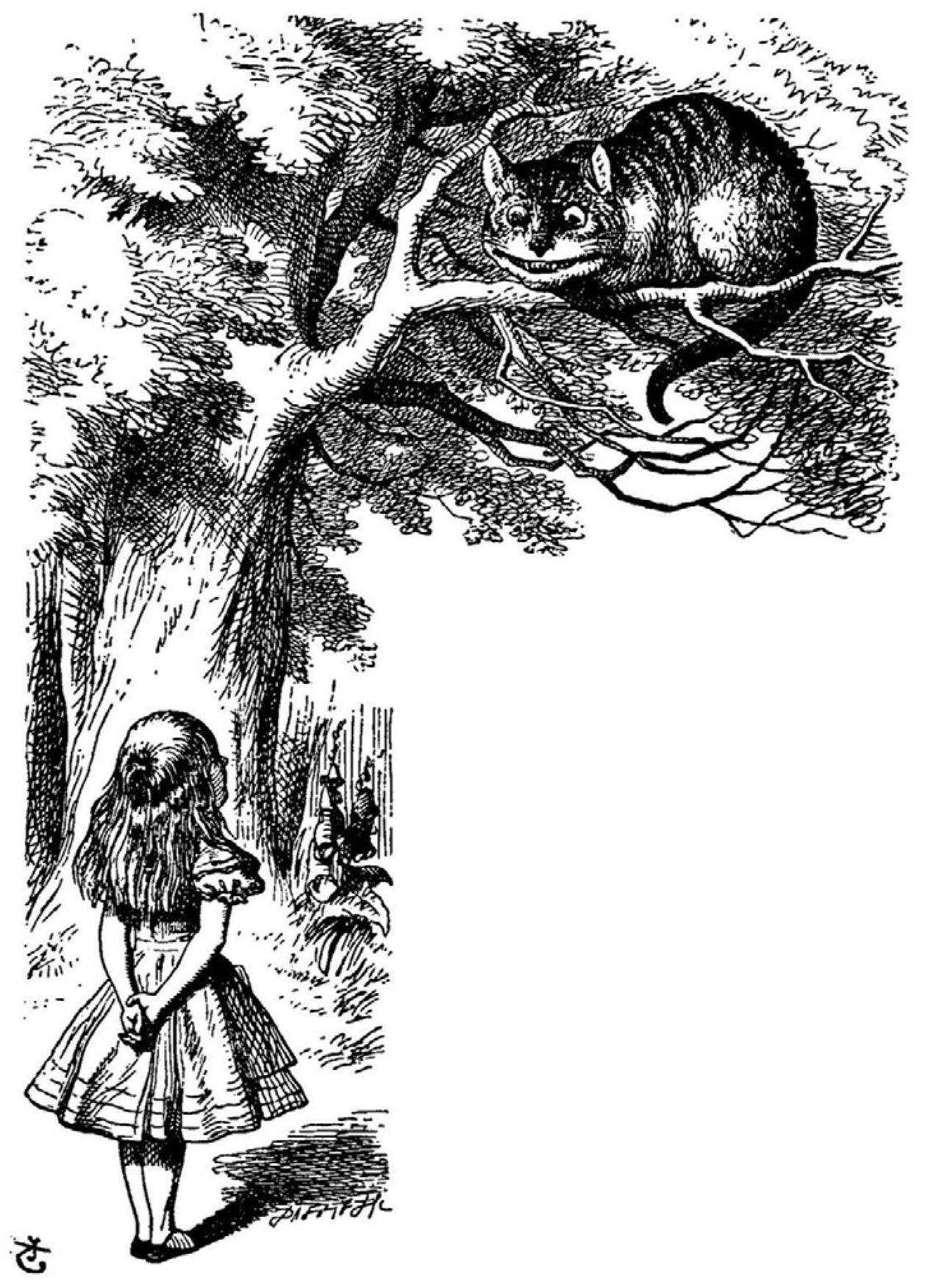

Nota: Esta ilustración está libre de Royalties. 
herentes y altamente estructurados. Así, afirma, que lo que probablemente hace eficaces las intervenciones es la correcta organización, planificación y coordinación del tratamiento, enfatizando la importancia de los espacios de elaboración por parte de los profesionales. Jugando con otro personaje del relato de Alicia en el País de las Maravillas, propone el veredicto del gato Cheshire para trastornos de personalidad, personaje que ya recomendó a Alicia: "siempre llegarás a alguna parte solo si caminas lo suficiente" para enfatizar la importancia de la intensidad y continuidad del tratamiento como elementos esenciales del cambio en este tipo de pacientes. Se hace necesario investigar en esta línea para comprender más en profundidad los mecanismos facilitadores del cambio en el trabajo con estos pacientes.

También resultan de interés las aportaciones de Livesly (2018) quien sugiere la utilización de modelos transdiagnósticos y transteóricos. En primera instancia, cuestiona los enfoques de diagnóstico específicos dados los problemas reflejados en los diagnósticos actuales. En segunda instancia, desde una perspectiva epistémica de corte posmoderno, sugiere un eclecticismo técnico que integre las intervenciones efectivas de los diferentes enfoques terapéuticos utilizados para el tratamiento de los trastornos de personalidad. Desde esta perspectiva, el enfoque integrativo sería pertinente para los trastornos de la personalidad dado que ofrecería una cobertura completa de los diferentes componentes de la patología de la personalidad y una mayor adecuación a cada caso individual (Livesly y Clarkin 2015; Livesly 2018).

Características personales del terapeuta. El trabajo con personas con un trastorno límite de la personalidad implica lidiar con contenidos de alto nivel emocional e importantes dificultades vinculares. Para los profesionales, su propio estilo relacional y las emociones sentidas son una herramienta muy útil de trabajo, pero también pueden ser una fuente de iatrogenia. Las características personales del terapeuta pueden ayudar a conseguir los resultados deseados, pero los posibles rasgos patológicos pueden entorpecer el trabajo psicoterapéutico. Las reacciones narcisistas del terapeuta como fantasías salvadoras, de poder, sádicas o manipuladoras bloquean toda posibilidad de avance.

Los pacientes con un trastorno de personalidad grave generan importantes dificultades contratransferenciales y a menudo ponen de manifiesto las limitaciones del estilo relacional del profesional. Estos pacientes suelen llevar al terapeuta a entrar en contacto con sus partes menos logradas y con los puntos más dolorosos de su propia personalidad. Los terapeutas son en sí mismos su propio instrumento de trabajo y si existen importantes dificultades o necesidades patológicamente satisfechas con el trabajo, la ayuda a estos pacientes será difícil (Rodríguez Cahill, 2015)

Los pacientes diagnosticados con un trastorno límite de la personalidad a menudo presentan esquemas rígidos, extremos y una forma de percepción que entra en colusión con las características de algunos psicoterapeutas. La adherencia extrema y la rigidez en la aplicación de técnicas por parte de los terapeutas puede constituir un componente potencialmente dañino. Así el psicoterapeuta debe mantener una actitud abierta y un uso flexible de las herramientas terapéuticas (Castonguay et al., 2010). No es sorprendente que bajos niveles de empatía y calidez del terapeuta, ya sean evaluados por los pacientes o por los propios terapeutas, son buenos predictores del posible deterioro en el paciente (Mohr, 1995). También la omnipotencia terapéutica, el retraimiento emocional o el sometimiento al paciente desde una total dedicación supone asumir posiciones muy inadecuadas para llevar a cabo el tratamiento. Otro factor de posible iatrogenia proviene de la disonancia resultante entre la experiencia interna del paciente y la perspectiva del terapeuta, a veces sentida como impuesta. Esto puede provocar desconcierto e incluso una mayor inestabilidad en el paciente (Fonagy y Bateman, 2006). Razón por la cual se aboga por la co-construcción de la formulación de las dificultades de los pacientes como sugieren Fonagy y Bateman (2017) desde la terapia basada en la mentalización.

Por último, las personas con un trastorno límite de la personalidad plantean un reto especial con respecto a los límites en la práctica profesional. Especialmente las personas con un trastorno límite grave presentan una tendencia a la transgresión del encuadre y ponen a prueba los límites de la relación terapéutica. Por tanto, la explicitación y el mantenimiento de las condiciones del encuadre son especialmente importantes. La puesta a prueba de los límites del encuadre es habitual y esperable por parte de los pacientes, pero iatrogénica cuando sucede por parte del terapeuta, quien debe evitar que sus necesidades, dificultades o intereses económicos o 
personales predominen por encima de la ayuda al paciente (Rodríguez Cahill, 2015). Gabbard (1994a, 1994b), experto en violaciones de los límites por parte de terapeutas, señala que la más grave de las transgresiones, el mantenimiento de relaciones sexuales entre terapeuta y paciente, tiene de base habitualmente rasgos de narcisismo patológico en el terapeuta.

El trabajo personal del terapeuta, los espacios de reflexión en los equipos y las supervisiones permiten minimizar el impacto de este factor iatrogénico.

Manejo de la alianza terapéutica y de la contratransferencia. Si la terapia es una interacción dialéctica relacional entre terapeuta y paciente, el mayor riesgo perjudicial lo tiene justamente la propia relación terapéutica, a la vez que supone un gran potencial de cambio (Rodríguez Cahill, 2015). La alianza terapéutica podría definirse como una construcción conjunta entre paciente y terapeuta fundamentada en el intercambio de opiniones y expectativas que dan paso a un proceso de objetivos conjuntos. La mayoría de los psicoterapeutas se han convencido de que la alianza terapéutica es el factor principal en el éxito de la misma, incluso por encima de la corriente psicológica que sustente nuestra práctica y que delimita la calidad del tratamiento que nuestros pacientes reciben. A propósito del modelo de Lambert, que estudia los factores generales de éxito en la terapia, los factores específicos y los porcentajes de cada uno de esos factores, los resultados reducen el impacto de las técnicas terapéuticas a un $15 \%$ del total (Assay y Lambert, 1999). No obstante, se ha de contemplar también que algunos autores consideran que la alianza terapéutica podría depender de otros factores además de la relación entre ambos y ser, por ejemplo, un efecto de la buena marcha del tratamiento (para una discusión, ver Brown, Mountford y Waller, 2013; también Martin, Garske y Davis, 2000, donde concluyen que la alianza terapéutica correlaciona $0,22 \mathrm{con}$ el resultado de la psicoterapia, por lo que apenas explicaría el 5\% del éxito terapéutico; Fluckinger et al., en su metaanálisis de 2018 concluyen que el impacto de la alianza sobre la varianza del resultado de la terapia ronda el 8\%; lo que demuestra que independientemente de las disquisiciones, la alianza terapéutica es un factor general relevante, pero en absoluto único).

En este sentido, la posibilidad de la ruptura de la alianza terapéutica enfrenta al profesional con la oportunidad de reparación del vínculo creado anteriormente. Para algunos autores la presencia inevitable de la ruptura se muestra como indicio de predicción de éxito terapéutico, ya que si se resuelve exitosamente podría dar lugar al restablecimiento de una nueva alianza más fortalecida (Baillargeon et al. 2012).

Safran y Muran (2000), así como Baillargeon y sus colaboradores (2012) identifican dos tipos de rupturas: por distanciamiento y por confrontación. En las primeras, el paciente toma distancia emocional, no solo del proceso terapéutico, sino también de sus propios procesos psíquicos y emocionales imposibilitando la transferencia en el proceso terapéutico. En la ruptura de la alianza terapéutica por confrontación el paciente muestra insatisfacción o enfado hacia el terapeuta o hacia el proceso de terapia. Los terapeutas deberán estar entrenados en estrategias metacomunicativas que ayuden a la reparación de la alianza terapéutica (Safran y Muran, 2000).

La relación terapéutica supone encontrar un equilibrio en términos de distancia y cercanía, aspecto especialmente difícil con los pacientes diagnosticados de trastorno de personalidad. En concreto, el paciente diagnosticado de trastorno límite de personalidad es altamente sensible en las relaciones de intimidad, debido a la estimulación de su sistema de apego. Estos pacientes, al presentar pobres habilidades mentalizadoras, se ven más expuestos a las actuaciones inadecuadas de los terapeutas. El profesional debe ser consciente de esta particularidad para evitar interacciones iatrogénicas (Bateman y Fonagy, 2006).

Además de la alianza terapéutica, el proceso de terapia discurre entre dos experiencias constantes en la interacción entre terapeuta y paciente: la transferencia y la contratransferencia. La transferencia, entendida como un vínculo a través del que se proyecta la realidad psíquica del paciente en el terapeuta, es un componente clave en los procesos psicoterapéuticos. Mientras que la contratransferencia sería la respuesta emocional global del terapeuta hacia el paciente tanto por aspectos personales de éste como en respuesta a sus proyecciones psíquicas. Los riesgos de este vínculo incluyen la omnipotencia y la omnisciencia del terapeuta y las fragilidades comparativas del paciente. Los lazos de apego a largo plazo con el terapeuta pueden hacer que la finalización de la terapia sea un evento muy difícil para estos pacientes, generando nuevos retos para el terapeuta en su relación con el paciente (Berk y Parker, 2009). 
Factores relacionados con el paciente. Aunque hay autores que se oponen a catalogar a algunos pacientes como difíciles por miedo a generar estigmatización y profecías auto-cumplidas (Duncan et al., 1997; Najavits, 2001), las investigaciones encuentran las siguientes características compartidas por los pacientes que son percibidos como difíciles por los terapeutas:

- Síntomas graves y prolongados (Mays y Franks, 1985; Wong et al., 1987).

- Baja motivación para el cambio y la terapia, locus de control externo respecto de su problema, o dificultad para la introspección y expresión. Estas características obstaculizarían el éxito de la mayoría de las intervenciones del terapeuta (Gold, 1995; Kottler, 1992; Powell, 1995).

- Dificultades graves para establecer y mantener relaciones interpersonales, lo que complica la relación terapéutica misma, además de asociarse con una falta de redes de apoyo social, aspecto predictor de un peor pronóstico (Clarkin y Levi, 2004; Mays y Franks, 1985; Truant, 1999).

Podríamos afirmar que estos pacientes difíciles obtienen poco o nulo beneficio de las intervenciones psicoterapéuticas por un profundo estado de desconfianza epistémica. En los últimos años, Bateman y Fonagy (2012) han incorporado a la teoría de la mentalización los hallazgos más recientes de investigaciones evolutivas tan relevantes como la teoría de la pedagogía natural de Csibra y Gergely $(2009,2011)$. Estos autores definen la confianza epistémica como la confianza en la autenticidad y relevancia de la información transmitida interpersonalmente. Desde estas nuevas aportaciones, la mentalización y el apego son entendidos como mediadores que garantizan la transmisión de conocimientos de una generación a otra, y los trastornos de personalidad son redefinidos como trastornos de la comunicación. La confianza epistémica puede ser dañada por la adversidad temprana. En la ausencia de confianza epistémica la capacidad para el aprendizaje social y, por lo tanto, para el cambio, es muy complicada.

La teoría de la pedagogía natural considera que la comunicación humana está especialmente adaptada para transmitir conocimientos culturalmente relevantes entre los seres humanos y postula una adaptación mutua socio-cognitiva guiada por señales entre cuidador-bebé que aseguran esa transmisión. Csibra y Gergely $(2009,2011)$ proponen que los seres humanos hemos evolucionado para aprender acerca del conocimiento culturalmente transmitido y relevante, y esto sucede primeramente en el contexto de las relaciones de apego. La psicoterapia debería suponer una experiencia que permitiera la regeneración de la confianza epistémica con el objetivo de que el paciente pueda empezar a confiar en su mundo social como una fuente benigna de aprendizaje social. Por lo tanto, las intervenciones terapéuticas serán efectivas cuando posibiliten al paciente aprender de su entorno social (Rodríguez Cahill, 2015).

También cabe reseñar que la comorbilidad es un factor relevante a considerar en los pacientes con un trastorno de la personalidad pues complica el pronóstico, asociándose a una presentación sintomática más severa y a peores resultados del tratamiento según una revisión exhaustiva de Fonagy (2005).

Pensamos que para comprender los efectos de la comorbilidad se requiere de una mirada más honda. El concepto de comorbilidad parte de una concepción sumativa de problemas de salud mental. Consideramos de mayor utilidad una visión estructural que parte de una visión jerárquica, donde se concibe la identidad como una dimensión profunda y central a la hora de planificar el tratamiento en todos los pacientes. La evaluación de la personalidad es obligada en cualquier patología dado que si la patología identitaria está siendo infradiagnosticada será también infratratada. La patología de personalidad subyacente sin diagnosticar en pacientes con alteraciones de eje I, no solo es clave para el desenlace terapéutico, sino que implica un importante error diagnóstico y compromete las posibilidades terapéuticas.

Factores relacionados con el contexto. Dentro de este apartado queremos reflexionar sobre dos aspectos claves en el tratamiento integral de las personas con un diagnóstico de trastorno límite de la personalidad: el contexto institucional del terapeuta y el contexto familiar del paciente.

Resulta alarmante que, con demasiada frecuencia, no se aplican los tratamientos indicados ni se llevan a cabo en las condiciones requeridas por problemas relacionados con el contexto de atención de los profesionales que atienden al paciente. Cabe señalar una diferencia fundamental: el tratamiento o abordaje general de un paciente no es lo mismo que una psicoterapia. Especialmente en el trabajo con pacientes graves, el tratamiento óptimo es un abordaje en red, planificado a largo plazo, intensivo, secuenciado 
en etapas y llevado a cabo por un equipo coordinado (Melendo y Cabrera, 2008). Una de las principales fuentes de iatrogenia con pacientes con patología identitaria grave es la ausencia de un plan de tratamiento, así como indicar un tratamiento en un nivel asistencial no ajustado a las necesidades del paciente o llevar a cabo un tratamiento en condiciones no adecuadas, como sería realizar una psicoterapia con una baja frecuencia de sesiones.

Gunderson (2002) propone cuatro niveles asistenciales en orden de intensidad y grado de contención decreciente que permitirán un tratamiento integral del paciente: hospitalización completa, hospitalización parcial u hospital de día, tratamiento ambulatorio intensivo y el tratamiento ambulatorio. La mayoría de pacientes graves necesitan varios años de psicoterapia y diferentes modos de intervenciones para obtener mejorías a nivel de sus problemas relacionales y de su personalidad. Los diferentes tipos de intervenciones serán secuenciados o complementarios, pero siempre propuestos desde un plan general de tratamiento. Este autor señala que la piedra angular del tratamiento es la psicoterapia intensiva a nivel ambulatorio, nivel de referencia donde el paciente recibirá la mayor parte de su tratamiento. De especial utilidad será que un miembro del equipo asuma la función de coordinar y organizar el tratamiento.

Tabla 2. Niveles asistenciales propuestos por Gunderson (2002)

\section{NIVELES ASISTENCIALES PROPUESTOS POR GUNDERSON (2002)}

\begin{tabular}{|c|c|c|c|}
\hline IV & $\begin{array}{l}\text { Hospital } \\
\text { Medicación } \\
\text { Psicoeducación }\end{array}$ & $\begin{array}{l}\text { Manejo de la crisis } \\
\text { Evaluación } \\
\text { Plan de tratamiento }\end{array}$ & 2-10 días \\
\hline || & $\begin{array}{l}\text { Hospitalización } \\
\quad \text { parcial/residencial } \\
\text { Noche } \\
\text { Día }\end{array}$ & $\begin{array}{l}\text { Rehabilitación social } \\
\text { Habilidades para la vida cotidiana } \\
\text { Habilidades sociales } \\
\text { Construcción de una alianza (relacional) }\end{array}$ & $\begin{array}{l}2 \text { semanas }-3 \text { meses } \\
2 \text { semanas }-6 \text { meses } \\
2 \text { semanas }-6 \text { meses }\end{array}$ \\
\hline$\|$ & $\begin{array}{l}\text { Tratamiento ambulatorio intensivo } \\
\text { Grupo de autoevaluación } \\
\text { Manejo de casos } \\
\text { Terapia Conductual dialéctica } \\
\text { Prescripción familiar }\end{array}$ & $\begin{array}{l}\text { Adaptación social (conductual) } \\
\text { Socialización } \\
\text { Anticipación, planificación } \\
\text { Control de impulsos/emociones } \\
\text { Construcción de una alianza (relacional) }\end{array}$ & $\begin{array}{l}1-3 \text { meses } \\
6-18 \text { meses } \\
6-12 \text { meses } \\
2-6 \text { meses }\end{array}$ \\
\hline I & $\begin{array}{l}\text { Tratamiento ambulatorio } \\
\text { Prescripción de grupo } \\
\text { Psicoterapia }\end{array}$ & $\begin{array}{l}\text { Crecimiento psicológico } \\
\text { Habilidades interpersonales } \\
\text { Cambio intrapsíquico } \\
\text { Construcción de una alianza }\end{array}$ & $\begin{array}{l}1-2 \text { años } \\
1-6 \text { años } \\
6 \text { meses-4 años }\end{array}$ \\
\hline
\end{tabular}

La atención continuada institucional compone una experiencia emocional intensa para todos los pacientes y, a menos que se administre cuidadosamente, empeorará a los pacientes con trastornos graves de la personalidad al sobreestimular sus procesos de vinculación y apego. Esta sobreestimulación en el tratamiento puede explicar los malos resultados a largo plazo de algunos de estos pacientes que han sido tratados con tratamientos no adaptados (Bateman y Fonagy 2010). 
En relación con las dificultades derivadas del contexto familiar del paciente, Marsha Linehan (1993), desde su modelo biosocial, plantea una teoría dialéctica del desarrollo del trastorno límite de la personalidad, donde la vulnerabilidad biológica y un entorno invalidante interaccionan para la emergencia del trastorno. Los entornos invalidantes se caracterizan porque tienden a trivializar o castigar las expresiones emocionales, presentan simultáneamente un alto nivel de emocionalidad, no reconocen las necesidades emocionales y solo responden a expresiones emocionales extremas. En las personas con un trastorno límite, el aprendizaje de esta dinámica familiar, hace que suelan oscilar entre invalidarse o culparse a sí mismos por su sufrimiento emocional o bien señalar al resto de las personas como causantes de su malestar emocional. Para la autora estas personas presentan una pasividad activa, es decir, una manera de resolver sus problemas caracterizado por una actitud de depositación de la responsabilidad de sus emociones en los otros a través de la actuación. Linehan señala que un individuo vulnerable sometido a experiencias de invalidación desarrolla una disfunción en la regulación de emociones, característica central del trastorno (Melendo y Cabrera, 2010).

\section{Tabla 3.}

Pasividad activa Contexto invalidante

Búsqueda de soluciones en los demás Apariencia resolutiva

Las experiencias adversas tempranas, especialmente en relación al trauma de apego, son una condición esencial conocida y sólidamente investigada. La negligencia emocional, el maltrato físico, el abuso sexual, así como las perturbaciones en el apego, son factores etiológicos muy frecuentes. Las relaciones familiares se suelen caracterizar por ser conflictivas, con alta expresividad emocional, límites intergeneracionales poco claros y una ausencia de estructura jerárquica adecuada, con escasa consistencia y durabilidad en los límites (Melendo y Cabrera, 2010). Obviamente, muchas familias pueden no entrar dentro de esta generalidad, pero sí es importante para el profesional evaluar las dinámicas familiares de los pacientes con los que trabaja (diagnóstico sistémico). La integración de la familia constituye una pieza clave para conseguir una evolución favorable en los pacientes con un trastorno límite de la personalidad. No ser considerados en el plan de tratamiento puede, no solo dificultar la mejoría del paciente, sino ser un daño para ellos al no ser atendido su sufrimiento y su malestar. El grado de la involucración de la familia en el tratamiento deberá ser valorado en cada caso particular, decidiendo el tipo de intervención a realizar entre una amplia gama: psicoeducación familiar individual o grupal, terapia de familia o pareja, grupos multifamiliares o un mero seguimiento.

\section{Gestión Iatrogénica}

Existe una gran evidencia de tratamientos efectivos para las personas con un diagnóstico de trastorno límite de la personalidad y existe sobrada literatura sobre las condiciones necesarias para su implementación. La terapia basada en la mentalización (MBT), la terapia dialéctico conductual (DBT) o la terapia focalizada en la transferencia (TFT) son ejemplos de tratamientos manualizados que han demostrado sobradamente su efectividad para el adecuado tratamiento de estos pacientes. Por lo tanto, la responsabilidad sobre el correcto abordaje de estos pacientes no solo implica a los clínicos sino también a los gestores de la salud mental y a los responsables políticos. Rodríguez Cahill (2015) define la gestión iatrogénica como los estilos y modos de gestión de algunos servicios de salud mental que dificultan la oferta de tratamientos indicados y en condiciones adecuadas para esta población, provocando, en consecuencia, un empeoramiento o cronificación de la patología. Como han demostrado ya varios estudios, cuando estos pacientes no reciben el tratamiento adecuado las 
consecuencias no implican solo un empeoramiento o cronificación de la patología, sino también un elevado coste económico para los servicios de salud (Salvador-Carulla et at., 2014; Bateman y Fonagy, 2013).

En los equipos de salud mental a menudo se encuentra una gestión de las agendas ineficaz, priorizando criterios económicos o políticos, basado en meros números o listas de espera, y no en criterios clínicos. Con frecuencia los equipos tienen espacios insuficientes de elaboración, de coordinación de casos y de supervisión. El trabajo de mentalización de los pacientes en el equipo es algo esencial en el trabajo con estos pacientes. Por desgracia, el tratamiento habitual que se oferta a estas personas son intervenciones psicológicas no especializadas a dosis infraterapéuticas y/o tratamiento estrictamente farmacológico. Es muy excepcional que las intervenciones se enmarquen dentro de un programa específico y que sea llevado a cabo por un equipo multidisciplinar que planifique el tratamiento de forma coherente, intensiva, con una estructura clara y bajo supervisión. Bateman y Fonagy (2004) señalan que el tratamiento de elección con pacientes con patología identitaria grave es el tratamiento en equipo. Estos autores, en el año 2000, hicieron una revisión de la bibliografía y concluyeron que lo que se había demostrado eficaz para el tratamiento del trastorno límite de personalidad tenía ciertas similitudes en la organización del tratamiento:

- Tratamientos con alto nivel de estructura.

- Implementados de forma consistente y confiable.

- Coherencia teórica.

- Tomaban en consideración las dificultades para establecer relaciones con estos pacientes, incluida la importancia de establecer una buena alianza terapéutica con el terapeuta y el equipo.

- Flexibilidad.

- Intensidad según la necesidad del paciente.

- Aproximación y cuidado personalizado.

- Buena integración y coordinación con otros servicios que atienden al paciente.

Se hace necesario una reorganización, tanto de las instituciones como de los equipos que trabajan con estos pacientes, para un correcto abordaje. Se ha visto que son útiles los programas específicos, los recursos socio-sanitarios con formación específica y los equipos suficientemente formados y supervisados (Melendo y Cabrera, 2008). Tratar en condiciones inadecuadas a estos pacientes tiene efectos nocivos sobre ellos y genera mayores costes a la sociedad. Modificar la cultura y el modo de trabajar de muchos centros pasa también por

Tabla 4. Factores de Iatrogenia

\section{FACTORES DE IATROGENIA}

\begin{tabular}{lll}
\hline TERAPEUTA & Concepto de TP & INTERVENCIONES IATROGÉNICAS \\
& Evaluación clínica & \\
& Intervención & \\
& Características personales & \\
& Manejo de alianza terapéutica y contratranferencia & \\
\hline PACIENTE & Síntomas graves y prolongados & FACTORES RIESGO IATROGENIA \\
& Baja motivación, locus control externo o dificultad para introspección \\
& Dificultades en establecimiento y mantenimiento relaciones. Falta de red social & \\
\hline CONTEXTO & Contexto de atención, derivación y coordinación de la red profesional & GESTIÓN IATROGÉNICA \\
\hline
\end{tabular}


un cambio en el estilo de gestión, que solo es posible con el compromiso de puestos de dirección, gerencias, servicios centrales y políticos. Por lo tanto, organizar de manera eficiente las intervenciones con estos pacientes es una necesidad de primer orden en la práctica de los servicios de salud mental, tanto en el ámbito público como privado, por cuestiones éticas, de salud y económicas.

\section{La prevención de la iatrogenia}

La prevención de la iatrogenia responde al primer principio ético de no maleficiencia que formuló Hipócrates con su primum non nocere. Actualmente se conoce como prevención cuaternaria. Marc Jamoulle (1986), médico belga, propuso el concepto de prevención cuaternaria para definir la intervención que evita o atenúa las consecuencias de la actividad innecesaria o dañina por parte del sistema sanitario. En el campo de la salud mental, la sobrevaloración de nuestras capacidades preventivas, diagnósticas o terapéuticas, así como nuestras necesidades personales de tener siempre respuesta a todo o de ser incapaces de decepcionar a los pacientes que nos piden una solución, nos lleva a intervenir de forma innecesaria, inadecuada y excesiva (Ortiz e Ibañez, 2012).

La psicoterapia depende, en gran medida, del modo en que el psicoterapeuta o el equipo terapéutico ponen en acción los componentes de la misma. Así, a diferencia de lo que suele ocurrir en el campo médico, donde se siguen protocolos con precisión, en psicoterapia se produce una enorme variabilidad en la utilización de los procedimientos, duración del tratamiento y otras variables que inciden en los resultados (Royal College of Psychiatrists, 2013). Una de las posibles ideas para mejorar la referencia a resultados iatrogénicos que pueden ser producidos por alguna de las variables intervinientes durante el proceso terapéutico, consiste en normalizar el lenguaje a la hora de hablar de consecuencias perjudiciales para el paciente. Parry (2016) propone que se empleen tres categorías que ayudarían en la investigación: una que recoja los episodios adversos que suceden durante el tratamiento o inmediatamente después de su finalización, como pueden ser intentos autolíticos, ingresos o suicidio del paciente; una segunda categoría que contemple el deterioro mental clínicamente significativo, como puede ser la aparición de nuevos síntomas y la tercera categoría contemplaría la experiencia subjetiva negativa del paciente atribuible a la terapia, aunque no existan elementos que se recojan en las categorías anteriores; aquí se incluirá que el paciente experimente ese daño fruto de la intervención terapéutica o que otras personas de su entorno puedan experimentar daño por motivo del tratamiento. Los autores consideran de especial relevancia este tipo de información, para permitir aquilatar las conclusiones que surgen de los metaanálisis, solicitando un mayor nivel de detalle en cuanto a las evidencias de daño y efectos indeseados atribuibles a las variables de la intervención psicoterapéutica (Parry et al, 2016). Mucho trabajo queda por hacer antes de consolidar la investigación en este campo.

\section{Recomendaciones para la prevención de la iatrogenia en pacientes con un trastorno de personalidad}

\section{Voluntades Anticipadas / Consentimientos Informados}

A partir de la aprobación de la Convención de los Derechos de las Personas con Discapacidad (CDPD) (Organización de las Naciones Unidas [ONU], 2006), se han identificado situaciones de vulnerabilidad en salud mental y se consideran los documentos de voluntades anticipadas como uno de los procedimientos para garantizar que se respeten los derechos personales en materia de tratamiento. Schwend et al. (2016) señalan que existen evidencias a propósito de las mejoras que producen este tipo de iniciativas en cuanto a: empoderamiento, autoconcepto, incremento de la autoestima, incremento de la participación en el proceso de toma de decisiones, responsabilidad sobre el proceso de la enfermedad, manejo de síntomas, control del propio tratamiento y congruencia entre valores y preferencias.

Otro efecto importante mencionado en diversos artículos es el aumento de la conciencia de enfermedad, la mejora en la prevención, identificación y tratamiento de las recaídas, así como la existencia de un plan de 
crisis a emplear de ser necesario. También se destacan las mejoras en cuanto a la adherencia al tratamiento e incremento de la confianza en los servicios de salud mental. Uno de los problemas que pueden encontrarse cuando se emplean modelos de toma de decisiones compartidas, pese a que son beneficiosas para el curso del tratamiento y el bienestar personal del paciente, estriba en que puede existir el prejuicio de que si alguien no puede adoptar decisiones en un ámbito [clínico], también será incapaz de tomarlas en otros relacionados (Ganzini et al., 2004, citado en Villagrán et al., 2014).

\section{Formación}

La psicoterapia es una opción clínico-asistencial que supone una alta cualificación por parte de los profesionales y de los equipos. Su prestación debe contar con las suficientes garantías de rigor científico y debe ponerse en marcha en condiciones adecuadas para su correcta implementación. El hecho de que las recomendaciones terapéuticas apuesten por la especialización de los profesionales introduce la necesidad perentoria de formalizar el entrenamiento en terapias manualizadas, pese a que existen algunas evidencias que apuntan a que algunas terapias sin apellido en el ámbito del tratamiento de trastornos de la personalidad también son efectivas (Bartak et al., 2010). Esto, probablemente, tenga relación con la eficacia de las variables comunes de los tratamientos empíricamente validados, algo que todavía necesita más investigación.

Una interesante estrategia de prevención consistiría en dar formación específica sobre iatrogenia en los planes formativos de grado, posgrado y formaciones especializadas de los profesionales de la salud mental. Un aspecto relevante que los terapeutas nóveles suelen echar de menos durante la formación es reforzar el trabajo de la persona del terapeuta y la formación práctica. También se ha visto que una gran mayoría de terapeutas consideran la supervisión como uno de los elementos más importantes en su desarrollo profesional y tener instancias de supervisión facilitadoras y contenedoras en su contexto laboral facilita la tarea (Orlinsky et al. 2005).

\section{Supervisión}

La supervisión es un elemento central en la práctica responsable de la psicoterapia, y un componente nuclear del tratamiento de pacientes con un trastorno límite de la personalidad. El mantenimiento de la función reflexiva del terapeuta y del equipo es clave para el éxito del tratamiento. Las supervisiones pueden dividirse en cuatro tipos con características y objetivos diferentes (Rodríguez Cahill, 2015):

- Supervisiones clínicas: La supervisión es una actividad teórico-práctica en el aprendizaje del quehacer psicoterapéutico y fundamental cuando se trabaja con pacientes especialmente complicados. Supone, además de una reflexión teórica y un desarrollo de las habilidades del terapeuta, un espacio para comprender las dificultades del profesional en su tarea, suponiendo para este un espacio de aprendizaje personal y de autocuidado.

- Supervisiones en equipo: El hecho de estar constituido como equipo de trabajo no implica necesariamente un funcionamiento como equipo terapéutico. Las supervisiones clínicas compartidas por todos los miembros del equipo permiten la comprensión y elaboración conjunta de las dificultades de los pacientes. Bateman y Fonagy (2004) plantean que el tratamiento debe realizarse bajo un modelo de equipo (one-team model) que dote de una estructura que permita contener las intensas proyecciones y minimice el riesgo de actuación de la contratransferencia por parte de los profesionales. La supervisión clínica en equipo permite realizar intervenciones coherentes y consistentes que redundarán en una mejoría de los pacientes.

- Supervisiones del equipo: Los equipos en salud mental se prestan a sí mismos como motor de transformaciones terapéuticas. El tratamiento a través del medio es la filosofía de tratamiento desde la que trabajan muchos equipos (hospitales de día, comunidades terapéuticas, centros de rehabilitación o unidades especializadas) y, por lo tanto, el peso de las dinámicas de los equipos son relevantes en los resultados terapéuticos. La supervisión de las dificultades surgidas en el trabajo en equipo por parte de sus miembros 
ayudará a minimizar el daño surgido por conflictos no elaborados del equipo, tanto para los pacientes como para los propios terapeutas.

- Supervisiones institucionales: Las organizaciones, como marco en el que se inscribe el trabajo de los terapeutas, son capaces de generar dinámicas muy potentes que influyen sobre los pacientes. La comprensión de los fenómenos institucionales es importante para evitar la iatrogenia causada por estos. La institución es un instrumento terapéutico, pero también puede ser un medio patógeno. La toxicidad de algunos factores institucionales repercute sobre el conocido síndrome del profesional quemado o burnout. Las supervisiones institucionales buscan comprender y movilizar las dinámicas organizacionales promoviendo unas relaciones más sanas entre los equipos y la institución.

\section{Evaluación del progreso y resultados de la terapia}

Un procedimiento que propuso Lambert (2004) consiste en evaluar antes y después de las sesiones el progreso del paciente, de tal modo que pueda considerarse si avanza al ritmo esperado, si existen mejoras respecto de lo previsible o si se produce algún deterioro como resultado de alguna de las variables moduladoras de la psicoterapia. En el estudio de Lo Coco (2008), donde también colaboró Lambert, concluyó que los cuatro factores que mejor evaluaban el progreso de los pacientes eran: empeoramiento general; síntomas de estrés (psicológico y quejas somáticas); relaciones interpersonales y rol social. Algunos instrumentos habituales en la evaluación del avance terapéutico son:

- Inventario WAI (Horvath y Greenberg, 1989) -Working Alliance Inventory- que permite evaluar los objetivos de la terapia, el trabajo terapéutico y la alianza entre ambos. En este inventario existe una forma para el terapeuta y otra para el paciente.

- BSL-23 (Bohus et al., 2009), es una versión reducida del cuestionario original que sirve para evaluar los síntomas en el trastorno límite y la gravedad de los mismos; es un buen instrumento para comprobar cuál es el grado de efectividad del proceso terapéutico, ya que al tratarse de un cuestionario que demanda poca dedicación por el número de ítems, se puede completar con cierta regularidad y a lo largo de toda la psicoterapia.

- PCOMS (Miller et al., 2005) -Partners for Change Outcome Management System-, incluye dos pruebas: ORS, -Outcome Rating Scale-, y SRS, -Session Rating Scale-. La ORS evalúa el funcionamiento del paciente en 4 áreas: bienestar individual, funcionamiento interpersonal, funcionamiento social y sensación general de bienestar; y la SRS evalúa la alianza terapéutica percibida por el paciente que muestra el vínculo entre paciente y terapeuta, el acuerdo en los objetivos de la psicoterapia, concordancia de las tareas, y la percepción general sobre la alianza.

- CORE-OM -Clinical Outcomes in Routine Evaluation-Outcome Measure-, es un instrumento creado por el Core System Group para la evaluación del cambio en el proceso terapéutico. Cuenta con una versión en español (Feixas et al., 2012) y consiste en un cuestionario que evalúa el estado psicológico del paciente a partir de cuatro dimensiones: Bienestar subjetivo, problemas/síntomas, funcionamiento general y riesgo. El CORE-OM destaca por su brevedad, versatilidad y por ser de acceso gratuito.

Estas herramientas permiten monitorizar el avance terapéutico e ir adoptando medidas que reduzcan los efectos iatrogénicos durante la intervención. Sumadas a las recomendaciones anteriores pueden facilitar la reducción de las dificultades originadas por el propio proceso terapéutico, para que el balance beneficios/perjuicios sea favorable para el paciente.

\section{Conclusiones}

Se hace necesario concienciar a los profesionales sobre la posibilidad de dañar a algunos pacientes como consecuencia de sus acciones o por omisión de estas. El fenómeno de la iatrogenia en psicoterapia es complejo y, en concreto, en el abordaje de las personas diagnosticadas de un trastorno límite de la personalidad 
requiere de mayor investigación. Desde la comunidad científica se debería empezar por establecer un consenso sobre lo que consideramos daño terapéutico y el mejor término a utilizar. Hemos propuesto hablar de iatrogenia para aludir a los efectos perjudiciales de los tratamientos y, a su vez, establecer una clasificación entre iatrogenia positiva (por acción) y negativa (por omisión). Cabe aclarar que el daño puede suceder en cualquier etapa del tratamiento, pero todavía no se ha conseguido especificar las variables concretas. El objetivo siempre debe buscar maximizar los efectos positivos de las intervenciones y minimizar los negativos, especialmente en los pacientes que nos ocupan, dado que el potencial efecto iatrogénico es mayor como consecuencia de la hiperactivación de su sistema de apego al comenzar una psicoterapia y de su profunda desconfianza epistémica.

Como recomendaciones generales para prevenir la iatrogenia con este tipo de pacientes, no solo estaría la formación en terapias empíricamente validadas, sino fundamentalmente la supervisión, el trabajo personal del terapeuta y la medición de la evolución de la terapia con instrumentos que permitan monitorizar posibles efectos adversos durante el proceso terapéutico. También es fundamental que estos pacientes reciban tratamientos intensivos, coherentes y consistentes pues tenemos suficientes datos para afirmar que estas características son un factor común de los tratamientos empíricamente validados para esta patología. Urge poner en marcha programas ambulatorios intensivos específicos para estos pacientes, nivel asistencial casi ausente en nuestro sistema sanitario, pero esencial para su tratamiento. Para terminar, queremos hacer hincapié en que consideramos la supervisión y la elaboración por parte de los equipos un elemento imprescindible del tratamiento de personas con un trastorno de la personalidad. Así corresponde a las instituciones y gerencias facilitar espacios para que esto tenga lugar.

Este artículo es el resultado del trabajo realizado por el grupo de trabajo "Trastornos de Personalidad" del Colegio Oficial de la Psicología de Madrid y pretende hacer reflexionar a los profesionales sobre el tipo de tratamientos que ofrecemos a estos pacientes. No supone una revisión sistemática de la bibliografía existente, pero sí recoge literatura relevante que reflejar algunos de los aspectos que hemos considerado potencialmente más dañinos para los pacientes con un trastorno límite de la personalidad. Sin duda todavía queda mucho que avanzar en el campo de la iatrogenia en psicoterapia y, en concreto, con pacientes con un trastorno de la personalidad.

\section{Referencias}

Asay, T. P. y Lambert, M. J. (1999). The empirical case for the common factors in therapy: Quantitative findings. En M. A. Hubble, B. L. Duncan y S. D. Miller (Eds.), The heart and soul of change: What works in therapy (1 ${ }^{\mathrm{a}}$ ed., pp. 23-55). American Psychological Association. https://doi.org/10.1037/11132-001

Bartak, A., Spreeuwenberg, M. D., Andrea, H., Holleman, L., Rijnierse, P., Rossum, B. V., Hamers, E. F., Meerman, A. M., Aerts, J., Busschbach, J. J., Verheul, R., Stijnen, T. y Emmelkamp, P. M. (2010). Effectiveness of different modalities of psychotherapeutic treatment for patients with cluster $\mathrm{C}$ personality disorders: results of a large prospective multicentre study. Psychotherapy and Psychosomatics, 79(1), 20-30. https:// doi.org/10.1159/000254902

Bateman, A. W. y Fonagy, P. (2004). Mentalization-Based Treatment of BPD. Journal of Personality Disorders, 18(1), 36-51. https://doi.org/10.1521/pedi.18.1.36.32772

Bateman, A. y Fonagy, P. (2006). Mentalizing and borderline personality disorder. En J. G. Allen y P. Fonagy (Eds.), The handbook of mentalization-based treatment (pp. 185-200). John Wiley y Sons Inc. https://doi. org/10.1002/9780470712986.ch9

Bateman, A. y Fonagy, P. (2010). Mentalization based treatment for borderline personality disorder. World Psychiatry: Official Journal of the World Psychiatric Association, 9(1), 11-15. https://doi.or$\mathrm{g} / 10.1002 / \mathrm{j} .2051-5545.2010 . t b 00255 . \mathrm{x}$

Bateman, A. y Fonagy, P. (Eds.). (2012). Handbook of mentalizing in mental health practice. American Psychiatric Publishing.

Bateman, A. y Fonagy, P. (2013). Mentalization Based Treatment. Journal of Psychoanalytic Inquiry, 33(6), 595-613. https://doi.org/10.1080/07351690.2013.835170 
Bateman, A., Campbell, C., Luyten, P. y Fonagy, P. (2017). A mentalization based approach to common factors in the treatment of borderline personality disorder. Current Opinion in Psychology, 21, 44-49. https://doi. org/10.1016/j.copsyc.2017.09.005

Bedics, J. D., Atkins, D. C., Harned, M. S. y Linehan, M. M. (2015). The therapeutic alliance as a predictor of outcome in dialectical behavior therapy versus nonbehavioral psychotherapy by experts for borderline personality disorder. Psychotherapy, 52(1), 67-77. https://doi.org/10.1037/a0038457

Baillargeon, P., Coté, R. y Douville, L. (2012). Resolution Process of Therapeutic Alliance Ruptures: A Review of the Literature. Psychology, 3(12), 1049-1058

Bergin, A. E. (1966). Some implications of psychotherapy for therapeutic practice. Journal of Abnormal Psychology, 71, 235-246. https://doi.org/10.1037/h0023577

Berk, M. y Parker, G. (2009). The Elephant on the Couch: Side-Effects of Psychotherapy. Australian y New Zealand Journal of Psychiatry, 43(9), 787-794. https://doi.org/10.1080/00048670903107559

Bohus, M., Kleindienst, N,. Stieglitz, R. D., Domsalla, M., Chapman, A. L., Steil, R., Philipsen, A. y Wolf, M. (2009). The short version of the borderline symptom list (BSL 23): Development and initial data on psychometric properties. Psychopathology, 42(1), 32-39. https://doi.org/10.1159/000173701

Boisvert, C. M. y Faust, D. (2002). Iatrogenic symptoms in Psychotherapy: A theoretical exploration of the potential impact of labels, language, and belief systems. American Journal of Psychotherapy, 56(2), 244-259. https://doi.org/10.1176/appi.psychotherapy.2002.56.2.244

Brown, A., Mountford, V.A. y Waller, G. (2013). is the therapeutic alliance overvalued in the treatment of eating disorders? International Journal of Eating Disorders, 46(8), 779-782. https://doi.org/10.1002/eat.22177 Carulla, L. S., Lucas, R., Ayuso-Mateos, J. L. y Miret, M. (2014). Use of the term Wellbeing and Quality of life in health sciences: A conceptual framework. The European Journal of Psychiatry, 28(1), 50-65. https:// doi.org/10.4321/s0213-61632014000100005

Castonguay, L. G., Boswell, J. F., Constantino, M. J., Goldfried, M. R. y Hill, C. E. (2010). Training implications of harmful effects of psychological treatments. American Psychologist, 65(1), 34-49. https://doi. org/10.1037/a0017330

Chartonas, D., Kyratsous, M., Dracass, S., Lee, T. y Bhui, K. (2017). Personality disorder: still the patients psychiatrists dislike? The Psychiatric Bulletin, 41(1), 12-17. https://doi.org/10.1192/pb.bp.115.052456

Clarkin, J. y Levi, K. (2004). The influence of client variables on psychotherapy. En M. Lambert (Ed.), Bergin and Garfield's handbook of psychotherapy and behavior change. Wiley and Sons.

Coyle, T. N., Shaver, J. A. y Linehan, M. M. (2018). On the potential for iatrogenic effects of psychiatric crisis services: The example of dialectical behavior therapy for adult women with borderline personality disorder. Journal of consulting and clinical psychology, 86(2), 116-124. https://doi.org/10.1037/ccp0000275

Crawford, M. J., Thana, L., Farquharson, L., Palmer, L., Hancock, E., Bassett, P., Clarke, J. y Parry, G. D. (2016). Patient experience of negative effects of psychological treatment: results of a national survey. The British journal of psychiatry: the journal of mental science, 208(3), 260-265. https://doi.org/10.1192/bjp. bp.114.162628

Csibra, G. y Gergely, G. (2001). Natural pedagogy as evolutionary adaptation. Philosophical Transactions of the Royal Society of London, 366(1567), 1149-1157. https://doi.org/10.1098\%2Frstb.2010.0319

Csibra, G. y Gergely, G. (2009). Natural pedagogy. Trends in Cognitive Sciences, 13(4), 148-153. https://doi. org/10.1016/j.tics.2009.01.005

Eysenck, H. J. (1965). The effects of psychotherapy. International Journal of Psychiatry, 1, 97-178.

Feixas, G., Evans, C., Trujillo, A., Saúl, L. A., Botella, L., Corbella, S., González, E, Bados, A., García Grau, E. y López-González, M. A. (2012). La versión española del CORE-OM: Clinical Outcomes in Routine Evaluation - Outcome Measure. Revista de Psicoterapia, 23(89), 109-135. https://doi.org/10.33898/rdp. v23i89.641

Fonagy, P., Roth, A. y Higgitt, A. (2005). Psychodynamic psychotherapies: Evidence-based practice and clinical wisdom. Bulletin of the Menninger Clinic, 69(1) 1-58. https://doi.org/10.1521/bumc.69.1.1.62267

Fonagy, P. y Bateman, A. (2006). Progress in the treatment of borderline personality disorder. The British journal of psychiatry, 188(1), 1-3. https://doi.org/10.1176/appi.psy.44.3.237 
Fonagy, P. y Allison, E. (2014). The role of mentalizing and epistemic trust in the therapeutic relationship. Psychotherapy, 51(3), 372-380. https://doi.org/10.1037/a0036505

Flückiger, C., Del Re, A. C., Wampold, B. E. y Horvath, A. O. (2018). The alliance in adult psychotherapy: A meta-analytic synthesis. Psychotherapy, 55(4), 316-340. https://doi.org/10.1037/pst0000172

Gabbard, G. O. (1994a). Psychotherapists who transgress sexual boundaries with patients. Bulletin of the Menninger Clinic, 58(1), 124-135.

Gabbard, G. O. (1994b). Sexual misconduct. Review of Psychiatry, 13, 433-456.

Ganzini, L., Volicer, L., Nelson, W. A., Fox, E. y Derse, A. R. (2004). Ten myths about decision-making capacity. Journal of the American Medical Directors Association, 5(4), 263-267. https://doi.org/10.1097/01. jam.0000129821.34622.a2

Gold, J. (1995). Knowing and not knowing: commentary on the roots of psychotherapeutic failure. Journal of Psychotherapy Integration, 5(2), 167-170. https://doi.org/10.1037/h0101215

Gunderson, J. G. y Gabbard, G. O. (Comps.). (2002). Psicoterapia en los trastornos de personalidad. Ars Medica.

Horvath, A. O. y Greenberg, L. S. (1989). Development and Validation of the Working Alliance Inventory. Journal of Counselling Psychology, 36(2), 223-233. https://doi.org/10.1037/0022-0167.36.2.223

Hubble, M. A., Duncan, B. L. y Miller, S. D. (Eds.). (1999). The heart and soul of change: What works in therapy. American Psychological Association.

Infocop. (2013). Graves deficiencias en la atención a la salud mental, informe del Defensor del Pueblo Andaluz. Infocop Online. http://www.infocop.es/view article.asp?id=4520

Jamoulle, M. (1986). Information et Informatisation en Medecine Generale. En J. Berleur, Lobet-Maris, C., R. F. Poswick y G. Valenduc (Eds.), Actes des III Journées de Réflexion sur l'Informatique ( $\left.3^{\circ} J . R . I.\right)$ (pp. 193-209). Presses Universitaires de Namur.

Jarrett, C. (2008). When therapy causes harm. The Psychologist, 21, 10-12. https://thepsychologist.bps.org.uk/ volume-21/edition-1/when-therapy-causes-harm

Jones, L. F. (2007). Iatrogenic interventions with personality disordered offenders. Psychology, Crime and Law, 13(1), 69-79. https://doi.org/10.1080/10683160600869809

Kottler, J. (1992). Compassionate therapy: working with difficult clients. Jossey-Bass Publishers.

Lambert, M. J. y Barley, D. E. (2002). Research summary on the therapeutic relationship and psychotherapy outcome. En J. C. Norcross (Ed.), Psychotherapy relationships that work (pp. 17-32). Oxford University Press.

Lambert, M. J., Gregersen, A. T. y Burlingame, G. M. (2004). The Outcome Questionnaire-45. En M. E. Maruish (Ed.), The use of psychological testing for treatment planning and outcomes assessment: Instruments for adults ( $3^{\mathrm{a}}$ ed., pp. 191-234). Lawrence Erlbaum Associates Publishers.

Lieb, K., Zanarinim M. C., Schmahl, C., Linehan, M. M. y Bohus, M. (2004). Borderline personality disorder. The Lancet, 364(9432), 453-461. https://doi.org/10.1016/S0140-6736(04)16770-6

Linehan, M.M. (1993). Diagnosis and treatment of mental disorders. Cognitive-behavioral treatment of borderline personality disorder. Guilford Press.

Livesley, W. J. (2018). Terapia Transdiagnóstica y Transteórica para el Trastorno de Personalidad. [Transdiagnostic and Trans-theoretical therapy for personality disorder]. Revista de Psicoterapia, 29(110), 89- 110.

Livesley, W. J. y Clarkin, J. F., (2015). Diagnosis and assessment. En W. J. Livesley, G. Diaggio y J. F. Clarkin (Eds.), Integrated treatment for personality disorder: A modular approach (pp. 51-79). Guilford Press.

Lo Coco G., Chiapelli M., Bensi L., Gullo S., Prestano C. y Lambert M. J., (2008). The Factorial Structure of the Outcome Questionnaire-45: A Study with an Italian Sample. Clinical Psychology and Psychotherapy, 15(6), 418-423. https://doi.org/10.1002/cpp.601

Martin, D. J., Garske, J. P. y Davis, M. K. (2000). Relation of the therapeutic alliance with outcome and other variables: A meta-analytic review. Journal of Consulting and Clinical Psychology, 68(3), 438-450. https:// doi.org/10.1037/0022-006x.68.3.438

Mays, D. T. y Franks C. M. (1985). Negative Outcome in Psychotherapy and What to do About It. Springer Publishing. 
Melendo Granados, J. J. y Cabrera Ortega, C. (2008). Trastorno límite de la personalidad. Guía para el profesional. Servicio Madrileño de Salud.

Miller, S. L., Duncan, B. L., Sorrell, R. y Brown, G. S. (2005). The Partners for Change Outcome Management System. Journal of Clinical Psychology, 61, 199-208.

Mohr, D. C. (1995). Negative outcome in psychotherapy: A critical review. Clinical Psychology: Science and Practice, 2(1), 1-27. https://doi.org/10.1111/j.1468-2850.1995.tb00022.x

Najavits, L. M. (2001). Helping 'difficult' patients. Psychotherapy Research, 11(2), 131-152. https://doi. org/10.1093/ptr/11.2.131

Organización de las Naciones Unidas (ONU). (2006). Convención sobre los derechos de las personas con discapacidad. www.un.org/esa/socdev/enable/documents/tccconvs.pdf

Orlinsky, D. E., Rønnestad, M. H. y Collaborative Research Network of the Society for Psychotherapy Research. (2005). How psychotherapists develop: A study of therapeutic work and professional growth. American Psychological Association. https://doi.org/10.1037/11157-000

Ortiz-Lobo, A. y Ibáñez-Rojo, V. (2011). Iatrogenia y prevención cuaternaria en salud mental. Revista Española Salud Pública, 85(6), 513-523.

Parry, G. D., Crawford, M. J. y Duggan, C. (2016). Iatrogenic harm from psychological therapies - time to move on. The British Journal of Psychiatry, 208, 210-212. https://doi.org/10.1192/bjp.bp.115.163618

Powell, D. (1995). Lessons learned from therapeutic failure. Journal of Psychotherapy Integration, 5(2), 175-182.

Rodríguez Cahill, C. (2015). Los desafíos de los trastornos de la personalidad (salud mental colectiva). Ed. Grupo 5.

Roth A. y Fonagy P. (2004). What Works for Whom? (2nd edition). Guilford Publications, Inc.

Royal College of Psychiatrists (2013). Report of the Second Round of the National Audit of Psychological Therapies (NAPT). Healthcare Quality Improvement Partnership.

Ruiz, A. (2019). Terapias potencialmente peligrosas y otras variables que producen iatrogenia en psicoterapia. Universidad Pontificia de Comillas. https://repositorio.comillas.edu/xmlui/bitstream/handle/11531/31778/ TFGTPP-RuizPantoja\%2C\%20Adriana.pdf? sequence=1 \&isAllowed=y

Sánchez Becerril, F. (25 de febrero de 2019). El caos de los psicólogos en la pública: por qué la mayoría de la gente va a privados. El Español. https://cutt.ly/CRA6yaM

Safran, J. D. y Muran, J. C. (2000). Resolving therapeutic alliance ruptures: Diversity and integration. Journal of Clinical Psychology, 56(2), 233-243. https://doi.org/10.1002/(SICI)1097-4679(200002)56:2<233::AIDJCLP9>3.0.CO:2-3

Semerari, D. y Dimaggio, G. (2008). Los trastornos de personalidad. Modelos y tratamiento. Desclée De Brouwer. https://doi.org/10.1002/cpp.555

Sinnaeve, R., Van Den Bosch, L., Hakkaart-van Roijen, L., Vansteelandt, K. (2018). Effectiveness of stepdown versus outpatient dialectical behaviour therapy for patients with severe levels of borderline personality disorder: a pragmatic randomized controlled trial. Borderline Personality Disorder and Emotion Dysregulation, 5, 12. https://doi.org/10.1186/s40479-018-0089-5

Suess Schwend, A, Bono Del Trigo, A., Ibáñez Rojo, V., Romero Cuesta, J., Romero Jimeno, M. D., Tamayo-Velázquez, M. I. y Huizing, E. (2016). Planificación Anticipada de Decisiones en Salud Mental: modelos, utilidades y propuestas de aplicación. Revista de la Asociación Española de Neuropsiquiatría, 36(129), 79-102.

Timimi, S. (2014). No more psychiatric labels: Why formal psychiatric diagnostic systems shoul be abolished. International Journal of Clinical and Health Psychology, 14(3), 208-215. https://doi.org/10.1016/j. ijchp.2014.03.004

Truant G. S. (1999). Assessment of suitability for psychotherapy. American Journal of Psychotherapy, 53(1), 17. https://doi.org/10.1176/appi.psychotherapy.1999.53.1.17

Van der Hart O., Nijenhuis, E. R. S., Steele, K. (2008). El yo atormentado. La disociación estructural y el tratamiento de la disociación crónica. Desclée de Brouwer.

Villagrán, J. M., Ruiz-Granados, L. y González-Saiz, F. (2014). El proceso de decisión compartida en el tratamiento del paciente psiquiátrico: estudios empíricos y evaluación de la capacidad. Revista de la Asociación Española de Neuropsiquiatría, 34(123), 491-506. 
Wong, S. E., Terranova, M. D., Bowen, L, Zarate, R, Massel, H. K. y Liberman, R. P. (1987). Providing independent recreational activities to reduce stereotypic vocalizations in chronic schizophrenics. Journal of Applied Behavior Analysis, 20(1), 77-81. https://doi.org/10.1901/jaba.1987.20-77

Zanarini, M. C. y Frankenburg, F. R. (2007). The Essential Nature of Borderline Psychopathology. Journal of Personality Disorders, 21(5), 518-535. https://doi.org/10.1521/pedi.2007.21.5.518

Artículo recibido: 07/09/2020

Artículo aceptado: 25/10/2021 